\title{
The New Direction of Senolytic Drug Research Should Be on Cancer Prevention, Adjuvant Therapies in Cancer and Healthy Aging in Cancer Survivors
}

Ozgur Tanriverdi ( $\nabla$ dr.ozgur.tanriverdi@gmail.com )

Mugla Sitki Kocman University Faculty Of Medicine, Department of Internal Medicine, Division of Medical Oncology, Mugla, Turkey https://orcid.org/0000-0002-0598-7284

Ummuhani Ozel Turkcu

Mugla Sitki Kocman Universitesi Tip Fakultesi

\section{Research Article}

Keywords: Aging, Cancer, Cellular senescence, Senolytics drugs

Posted Date: March 31st, 2021

DOI: https://doi.org/10.21203/rs.3.rs-307731/v1

License: (c) (i) This work is licensed under a Creative Commons Attribution 4.0 International License.

Read Full License 


\section{Abstract}

In cases where cellular senescence does not function properly, the use of drugs called senolytics in the prevention of chronic aging-related disorders such as cancer, diabetes and cardiovascular diseases has become a very interesting topic. There are studies showing that senolytic drugs can be used for the purpose of preventing cancer, preventing recurrence in individuals diagnosed with cancer, and delaying multimorbidity situations that may develop as long-term side effects of anti-cancer treatments. This article has been prepared with the aim of reminding general information about senolytic drugs and cellular senescence due to the fact that there are many controversial researches in the field of oncology in the future

\section{Introduction}

There are many hypotheses to illuminate the aging process [1]. The most widely accepted of these hypotheses can be listed as follows: Oxidative stress hypotheses and mitochondrial dysfunction hypothesis as well as genetic and epigenetic alterations mechanisms such as somatic mutations, Apolipoprotein E allele polymorphisms and telomere-telomerase activity defects [1].

Cellular senescence has an important place among these mechanisms and it is an interesting topic that is still intense research due to the difference of molecular and biochemical changes that occur during the health aging process [2].

Cancer is still the most important health problem today due to the aging world population [3]. Despite the dizzying advances in cancer treatments, it still ranks second among all causes of death after cardiovascular diseases [3]. The mechanisms of action of anti-cancer treatments are based on very different properties, and each treatment targets the elimination of cancer cells [4]. In fact, many anticancer drugs act by inducing aging in cancerous cells with different mechanisms [5]. Chemotherapeutics such as doxorubicin, etoposid, irinotecan, cisplatin, paclitaxel, bleomycin and temozolamide induce cellular aging by increasing SA- $\beta$-galactosidase enzyme activity or by causing upregulation of tumor suppressor genes such as p16, p21 [4,6-8]. Similar effects have been reported with cyclin-dependent kinase (CDK) 4/6 inhibitors, epigenetic modulators and molecular targeting monoclonal antibodies such as rituximab $[4,9,10]$. The cancer cell-destroying properties of these drugs may also have negative effects in cancer survivors by affecting the senescence process in normal tissues [4].

The researches of senolytic drugs on the destruction of senescent cells, especially those that have escaped from the healthy aging process, in the field of oncology that it is promising both prevent the development of cancer in healthy or high-risk individuals and increase the risk of inducing carcinogenesis by the emergence of new senescent cells in the follow-up after adjuvant treatments in patients with early stage cancer $[4,11]$. Achieving a healthy longevity is the main goal of senolytic drugs by eliminating not only carcinogenesis but also long-term side effects associated with cancer treatments, including 
radiotherapy, and preventing chronic aging-related patients that cause multiple morbidity in cancer survivors $[4,11]$.

This article has been prepared with the aim of reminding general information about senolytic drugs and cellular senescence due to the fact that there are many controversial researches in the field of oncology in the future.

\section{Cellular Senescence}

With aging, the risk of development of multiple chronic diseases, geriatric syndromes, impaired physical and cognitive functions, as well as associated mortality increases. There are many hypotheses regarding the basic senescence process. In these hypotheses, some molecular, physiological and biochemical changes in the basic aging process come to the fore, but there is no clear consensus mechanism yet [12].

In the senescence process, there is a chronic low-grade inflammation often accompanied by fibrosis. This is accompanied by functional disorders at the macromolecular level such as DNA damage, disruption in telomere activity, changes in repair mechanism and protein translation, lipotoxicity, and decreased autophagy. In addition, organelle dysfunction develops in the cells, especially at the mitochondrial level. It is known that dysfunction in the immune system and cellular aging accompany all of these [13].

Cellular aging was first reported in 1961 by Hayflick and Moorehead [14]. It involves a cellular mechanism that includes irreversible replicative arrest, sustained viability by resistance to apoptosis, and often increased metabolic activity. It is known that intracellular and extracellular signals that contribute to the cellular aging process are actually effective in tissue or cellular damage or cancer development $[12,13]$.

During the cellular senescence process, a number of proinflammatory cytokines, chemokines, proteases and other factors called aging-related secretory phenotype (SASP) may be secreted by aging cells. This condition contributes to aging and local and systemic dysfunction in a number of diseases $[12,13]$.

Some cells that are aging and display the SASP phenotype are called deleterious (D-) senescent cells and can make up $30-70 \%$ of the aging cell population. SASP components produced by D-senescent cells may differ depending on the aging cell type and the cause of aging. Factors released from these senescent cells that phenotypically show SASP can be listed as follows: 1) cytokines such as tumor necrosis factor- (TNF-) a, interleukin- (IL-) 6, IL-8 and others known to cause inflammatory, pro-apoptotic and insulin resistance, 2) Chemokines that play a role in cellular immunity, 3) Matrix metalloproteinases (MMP) such as MMP - 3, MMP - 9 and MMP - 12 that cause tissue damage, 4) Factors such as transforming growth factor- (TGF-) $\beta$ family members that contribute to stem cell and progenitor dysfunction as well as fibrosis development, 5) Activins and inhibins that induce stem cell and progenitor dysfunction as well as differentiation, 6) Serpins that can cause blood clotting and fibrosis, such as plasminogen activator inhibitor (PAI) - 1 and PAI - 2,7) Growth factors that induce tumor spread, 8) Bioactive lipids such as bradykinins, ceramides or prostaglandins that contribute to inflammation and tissue dysfunction, 9) Micro-RNAs (miRNAs) that play a role in inflammation and insulin resistance by 
contributing to stem cell and progenitor cell dysfunction, and 10) exosomes capable of transporting locally and systemically toxic and aging-causing cytotoxic metabolites $[5,12,14]$.

\section{Senolytics}

During the cellular senescence process, the first ideas about the prevention of chronic diseases and cancer, especially by eliminating D-senescent cells, came to the fore in the 2000s [15]. It has been shown that the accumulation of SA $\beta$ - gal in cells in animal studies has a negative effect on lifespan and stimulates the onset of age-dependent changes in many organs. Subsequent studies have reported that healthy aging can be prolonged in mice with reduced expression of p16Ink4a with calorie restriction or a mutation that decreases growth hormone signaling. These studies showed an inverse relationship between aging cell burden and duration of health [15]. Especially in the period after 2005, drug researches started with the aim of identifying fusion proteins for the recognition of senescent cells and killing Dsenescent cells. Studies related to these drugs, which are defined as senolytic drugs, have been based on the knowledge that aging cells are resistant to apoptosis [15].

Apoptosis resistance occurs despite the release of proapoptotic SASP factors from senescent cells. Therefore, hypothetically, anti-apoptotic mechanisms are required to overcome the resistance of aging cells to apoptotic stimuli $[15,16]$. After the researches, it has been reported that aging cells may have characteristics similar to cancer cells including apoptosis. As a result of this observation, it is concluded that senescent cells that secrete pro-apoptotic SASP can prevent their own extinction through protective senescent anti-apoptotic pathway (SCAP) networks. From proteomics and transcriptomic databases, SCAP pathways were found to be expressed at a higher rate in senescent cells [16].

Senolytic drugs work through different mechanisms to make the aging process healthier, especially by eliminating D-senescent cells. However, a senolytic drug that has a clear positive effect on this process has not been defined yet. However, promising research continues [16].

The first senolitics were discovered through the approach of identifying agents that temporarily disable SCAP networks that allow these cells to survive in the microenvironment created by D-senescent cells and kill the sensescent cells in their environment. From these drug studies, it has been reported that siRNAs, one of the small RNA types, have an effect against anti-apoptotic regulators in those related to the regulation of the anti-apoptotic process that is effective in selectively reducing aging cell viability, and this effect is dependent on the aging cell type $[5,15,16]$. The basis of this hypothetical approach is the research that is caused by siRNAs that reduce BCL-XL or other BCL-2 family members selectively to apoptosis of aging and decaying human umbilical vein endothelial primary cells (HUVECs) [16, 17]. This effect was proven to be selective, with the finding that they did not increase apoptosis induction against aging human primary adipocyte progenitor cells by the same siRNAs. Unfortunately, it has been reported that multiple SCAP pathways may need to be targeted to kill some senescent cells, and even antiapoptotic senolytics may be insufficient, as senescent cell defenses against apoptosis are far behind other SASP mechanisms in some senescent cells. Among the drug researches that provide a healthy 
aging process by eliminating D-senescent cells through SCAP networks, the most important ones are dasatinib, a tyrosine kinase inhibitor that targets JAK1 / 2, and quercetin, a natural flavonoid $[5,15,17]$. Unlike other tyrosine kinase inhibitors such as imatinib, which have been shown to be non-senolytic, dasatinib induces apoptosis caused by addiction receptors such as ephrins by partially inhibiting Src kinase. Fisetin, on the other hand, is another natural flavonoid that is considered senolytic with similar effects like dasatinib and quercetin [17]. Senolytic flavonoids such as quercetin and fisetin have been reported to act by partially inhibiting BCL-2 family members such as $B C L-x L$, as well as hypoxia-inducing factor (HIF)-1 $a$ and other SCAP network components $[17,18]$. In a study, it was determined that dasatinib targets senescent human cultured primary adipocyte progenitor cells, while quercetin destroys cultured HUVECs, interestingly, the combination of dansatinib and quercetin causes apoptosis of both senescent human primary adipocyte progenitor cells and senescent HUVECs $[5,15,17]$. However, sensescent adipocyte progenitor cells or HUVECs did not cause. However, due to the abundance of SCAP pathways, in some cell types such as mouse embryonic fibroblasts, neither dasatinib nor quercetin have been reported to be senolytic, while the combination of these two chemicals is senolytic [16-18]. All these studies show that the SASP phenotype and cell type are important in aging cells. However, due to the fact that the target of true senolytics is not a single receptor, enzyme or biochemical pathway, we may think that research may need to be advanced at the molecular and biochemical level. Indeed, agents such as navitoclax or Nutlin3a, a BCL-2 family member inhibitor that are defined as panolytic and act on single or limited targets, have significant non-target apoptotic effects on non-innate cell types such as platelets and the immune system. Also, nutlins can actually cause aging $(15,19,20)$.

\section{Conclusion}

As a result, the field of oncological research will expand with the use of currently known senolytic drugs such as dasatinib, quercetin, fisetin, navitoclax and nutlins in future studies to prevent cancer recurrence, as well as secondary cancers or multiple morbidity conditions that may be caused by the long-term side effects of anti-cancer drugs.

\section{Declarations}

Conflict of Interest: Author Ozgur Tanriverdi declares that he has no conflict of interest. Author Ummuhani Ozel Turkcu declares that she has no conflict of interest.

\section{Availability of data and material : None}

\section{Code availability: None}

Authors' contrubitions: Ozgur Tanriverdi (Thought and writing of the article, literature review and final reading) and Ummuhani Ozel Turkcu (Literature review and final reading).

Ethical approval: This article does not contain any studies with human participants or animals performed by any of the authors. 
Informed consent: Informed consent is not required as this article does not include any studies with human participants.

Consent for publication: Consent for publication is not required as this article does not include any studies with human participants.

\section{References}

1. Mc Auley MT, Guimera AM, Hodgson D, Mcdonald N, Mooney KM, Morgan AE, Proctor CJ (2017) Modelling the molecular mechanisms of aging. Biosci Rep 37: BSR20160177. https://doi.org/10.1042/ BSR20160177.

2. Weinert BT, Timiras PS (2003) Invited review: theories of aging. J Appl Physiol 95: 1706-1716. https://doi.org/10.1152/japplphysiol.00288.2003

3. Siegel RL, Miller KD, Jemal A (2020) Cancer statistics, 2020. CA Cancer J Clin 70: 7-30. https://doi.org/10.3322/caac.21590.

4. Wang B, Kohli J, Demaria M (2020) Senescent cells in cancer therapy: Friends or foes. Trends Cancer 6: 838-857. https://doi.org/j.trecan.2020.05.004

5. Chabner B, Roberts T (2005) Timeline: chemotherapy and the war on cancer. Nat Rev Cancer 5: 6572. https://doi.org/10.1038/nrc1529.

6. Yang M, Lin PM, Liu YC, Hsiao HH, Yang WC, Hsu JF, Hsu CM, Lin SF (2012) Induction of cellular senescence by doxorubicin is associated with upregulated mir-375 and induction of autophagy in K562 cells. PloS One 7: e37205. https://doi.org/journal.pone.0037205.

7. Roberson R, Kussick SJ, Vallieres E, Chen SYJ, Wu DY (2005) Escape from therapy-induced accelerated cellular senescence in p53-null lung cancer cells and in human lung cancers. Cancer Res 65: 2795-2803. https://doi.org/ 0008-5472.CAN-04-1270.

8. Wang X, Wong SC, Pan J, Tsao SW, Fung KH, Kwong DL, Sham JS, Nicholls JM (1998) Evidence of cisplatin-induced senescent-like growth arrest in nasopharyngeal carcinoma cells. Cancer Res 58: 5019-5022.

9. Hu W, Sung T, Jessen BA, Thibault S, Finkelstein MB, Khan NK, Sacaan Al (2016) Mechanistic investigation of bone marrow suppression associated with palbociclib and its differantiation from cytotoxic chemotherapies. Clin Cancer Res 22: 2000-2008. https://doi.org/ 078-0432.CCR-15-1421.

10. Dabritz JHM, Yu Y, Milanovic M, Schönlein M, Rosenfeldt MT, Dörr JR, Kaufmann AM, Dörken B, Schmitt CA (2016) CD20-targeting immunotherapy promotes cellular senescence in B-cell lymphoma. Mol Cancer Ther 15: 1074-1081. https://doi.org/ 10.1158/1535-7163.MCT-15-0627.

11. Mantovani A, Allavena P, Sica A, Balkwill F (2008) Cancer-related inflammation. Nature 454: 436-444. https://doi.org/10.1007/s10875-012-9847-0.

12. Wei W, Ji S (2018) Cellular senescence: Molecular mechanisms and pathogenicity. J Cell Physiol 233: 9121-9135. https://doi.org/10.1002/jcp.26956. 
13. Freund A, Patil CK, Campisi J (2011) P38MAPK is a novel DNA damage response-independent regulator of the senescence-associated secretory phenotype. EMBO J 30: 1536-1548. https://doi.org/10.1038/emboj.2011.69.

14. Hayflick L, Moorhead PS (1961) The serial cultivation of human diploid cell strains. Exp. Cell Res 25 : 585-621. https://doi.org/10.1016/0014-4827(61)90192-6.

15. Short S, Fielder E, Miwa S, von Zglinicki T (2019) Senolytics and senostatics as adjuvant tumour therapy. EbioMedicine 41: 683-692. https://doi.org/10.1016/j.ebiom.2019.01.056.

16. Kirkland JL, Tchkonia T, Zhu Y, Niedernhofer LJ, Robbins PD (2017) The clinical potential of senolytic drugs. J Am Geriatr Soc 65: 2297-2301. https://doi.org/10.1111/jgs.14969.

17. Zhu Y, Tchkonia T, Pirtskhavala T et al (2015) The Achilles' heel of senescent cells: from transcriptome to senolytic drugs. Aging Cell 14: 644-658. https://doi.org/10.1111/acel.12344.

18. Triantafyllou A, Mylonis I, Simos G, Bonanou S, Tsakalof A (2008) Flavonoids induce HIF-1 alpha but impair its nuclear accumulation and activity. Free Radic Biol Med 44: 657-670. https://doi.org/10.1016/j.freeradbiomed.2007.10.050.

19. Pal HC, Sharma S, Elmets CA, Athar M, Afaq F (2013) Fisetin inhibits growth, induces G(2)/M arrest and apoptosis of human epidermoid carcinoma A431 cells: role of mitochondrial membrane potential disruption and consequent caspases activation. Exp Dermatol 22: 470-475. https://doi.org/10.1111/exd.12181.

20. Zhu Y, Tchkonia T, Fuhrmann-Stroissnigg $\mathrm{H}$ et al (2016) Identification of a novel senolytic agent, navitoclax, targeting the Bcl-2 family of anti-apoptotic factors. Aging Cell 2016; 15: 428-435. https://doi.org/10.1111/acel.12445. 